\title{
Spatio-Temporal Spectrum Holes and the Secondary User
}

\author{
Martin B.H. Weiss, Member, IEEE
}

\begin{abstract}
DSA research must explicitly consider the perspective of secondary users. This paper considers the spatiotemporal properties of spectrum holes as they impact the secondary user's communications needs. Like Weiss et.al. [1], this paper develops a taxonomy of spectrum holes from the point of view of the secondary user. Each type of spectrum hole is analyzed for the kinds of communications requirements that can be supported, illustrated, where possible, by existing measurement data. The analysis concludes that a secondary user's ability to meet their communications need varies considerably. More detailed analysis of the spatio-temporal density of spectrum holes would be necessary to further quantify these conclusions.
\end{abstract}

Index Terms-Appropriate technology, Telecommunications, Radio Communications, Cognitive Radio

\section{INTRODUCTION}

$\mathrm{P}$ OINT-OF-VIEW matters in the analysis of dynamic spectrum access (DSA) systems. With some exceptions, DSA research has focussed on either general systems problems or problems that are analyzed largely from the perspective of the primary user. This is understandable because one must first have unused radio bands if one is to have DSA. More fundamentally, license holders are fewer in number (probably), have well defined applications and are motivated to ensure suitable QoS over their license tuple (space, time and frequency). In contrast, because secondary use is an emergent opportunity, potential secondary users' applications and goals are not well defined nor can they be easily identified, which means they cannot be easily organized into a credible interest group at a regulatory agency. Thus, it is not surprising that their viewpoint is not represented as fully in the regulatory debate. Nonetheless, secondary users are an essential part of the DSA picture, since there would be no DSA without them.

That is not to say that the secondary users have been ignored entirely, however. Weiss and Lehr [2] consider different strategies for DSA that explicitly consider secondary users. Akyildiz et.al. [3] consider user requirements as part of the spectrum analysis function, but do not elaborate. Chapin and Lehr [4] also consider user application factors, but again

Manuscript received November 15, 2010.

Martin BH Weiss is with the School of Information Sciences, University of Pittsburgh, Pittsburgh, PA 15260 USA. in general terms. This paper combines the spatio-temporal analysis of [1] with the generalized secondary user perspective adopted in [5] to determine feasible use cases for different kinds of spectrum holes.

To consider a secondary user's application requirements in more detail, it is important to consider both the spatial and temporal dimensions of spectrum holes [1,6]. Figure 1 illustrates the temporal characteristics at a single point in space $^{1}$. The vertical axis of this figure is time of day and the horizontal axis is frequency. If we assume that this figure contains a representative sample of any 24 hour period, one can see temporal spectrum holes (blue areas in the figure) with different characteristics. illustrates the spatial characteristics of spectrum [7], though it is a predicted coverage plot rather than a measured one and assumes a static signal. Here, the white areas would consist of spatial spectrum holes. As with Figure 1, one might infer spectrum holes with a variety of spatial characteristics.

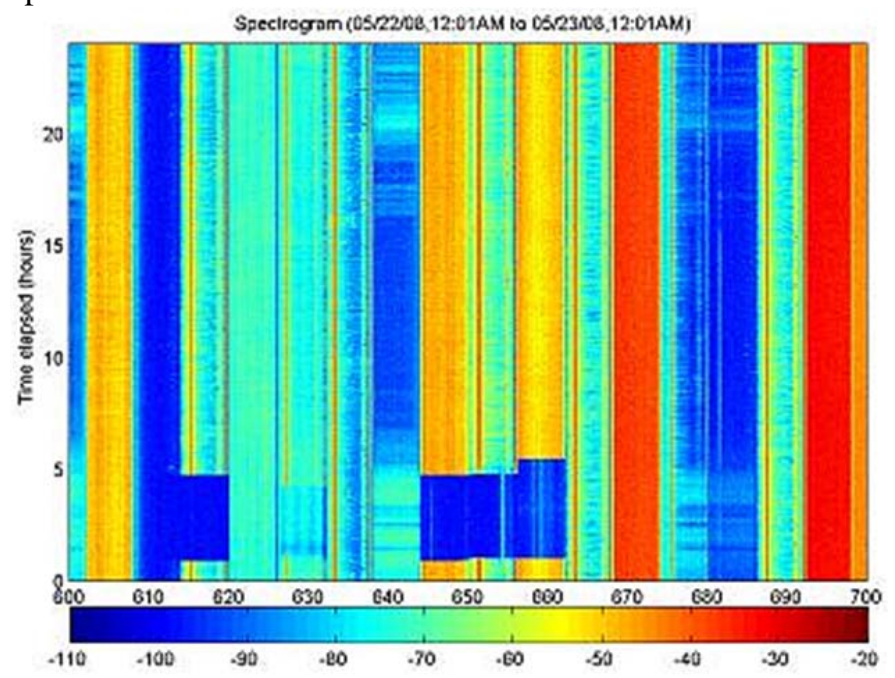

Figure 1 - Example of the temporal structure of spectrum

Weiss et.al. create a spatio-temporal taxonomy of spectrum holes by classifying their characteristics as static, periodic or stochastic in both time and space. Figure 3 combines these dimensions and provides some examples of representative real-world systems.

From the WINCOM lab at Illinois Institute of Technology (http://www.cs.iit.edu/ wincomweb/24hrtv.html). This figure illustrates the UHF television band (600-700 MHz) on the horizontal axis and time of day on the vertical axis. 
This paper follows a similar approach in that it examines how the spatio-temporal characteristics of spectrum holes affects the QoS experienced by potential secondary users. Tonmukayakul and Weiss [5] consider end user QoS in their paper, but the purpose of that paper was to study the conditions under which secondary use might occur (from the point of view of potential secondary users) rather than considering how the spatio-temporal characteristics of spectrum holes affect QoS. Since they were considering only cooperative secondary sharing, they could reasonably assume that the spectrum hole would be adequate as a result of the bargaining between primary and secondary users.

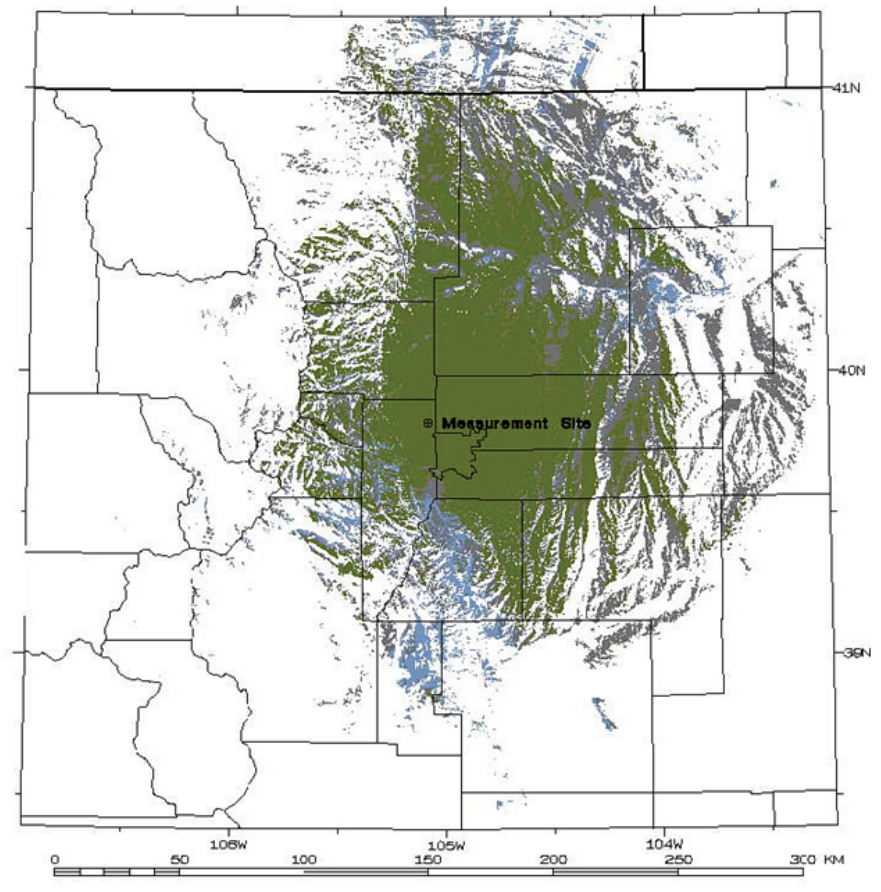

Figure 2 - Example of the spatial structure of spectrum

\begin{tabular}{|c|c|c|c|c|}
\hline & \multicolumn{3}{|c|}{ Spatial Characteristic } \\
\hline & & $\begin{array}{c}\text { Contig- } \\
\text { uous }\end{array}$ & Periodic & Stochastic \\
\hline \multirow{4}{*}{ 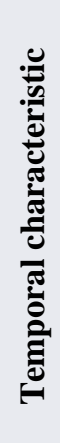 } & Static & $\begin{array}{l}\text { TV } \\
\text { White } \\
\text { Spaces }\end{array}$ & $\begin{array}{l}\text { Sensor } \\
\text { network }\end{array}$ & $\begin{array}{l}\text { CDMA } \\
\text { mobile }\end{array}$ \\
\hline & Periodic & $\begin{array}{l}\text { Daytime } \\
\text { broadcast }\end{array}$ & $\begin{array}{l}\text { Rotating } \\
\text { ant. Radar }\end{array}$ & - \\
\hline & $\begin{array}{l}\text { Fast } \\
\text { periodic }\end{array}$ & $\begin{array}{l}\text { LTE cell } \\
\text { site }\end{array}$ & - & $\begin{array}{l}\text { LTE } \\
\text { mobile }\end{array}$ \\
\hline & Stochastic & WiFi & - & $\begin{array}{l}\text { Public } \\
\text { safety }\end{array}$ \\
\hline
\end{tabular}

Figure 3 -- Taxonomy of spectrum holes based on primary user activity [1]

Modelling the spatial aspects of DSA-based systems has not received much attention from the research community thus far, even though it is generally recognized as a characteristic of spectrum holes [6]. Some researchers have made progress in addressing this gap in the research literature, however. In particular, [8] considers the spatial power spectral density using spatial statistics and [9] considers the spatial distribution of nodes in a communications system. Further, [10] begins to match the needs of primary and secondary users. Together, these contribute to an understanding of the spatial aspects of DSA as they apply to communications systems.

\section{Secondary Users' Perspective}

We begin by assuming that secondary users have a communications requirement that they seek to satisfy via a wireless channel. Paraphrasing [5], these users can choose an unlicensed band, secondary use, or commercial services. We further assume that these choices are ordered by net cost (which is the value of the communication less the cost of executing it). These choices also have potential quality tradeoffs; with unlicensed bands ordered by the variability of QoS (highest to lowest). A secondary user therefore must choose the best QoS for the net cost.

It is useful to be slightly more specific about QoS, since it depends on the nature of the communications requirement. Communications engineers have classified user communications requirements in several ways. One is to characterize them as "elastic" if they are tolerant of delay and delay variation and "inelastic" if they are not [11]. This relatively gross characterization is useful to an extent. However, additional parameters, such as absolute delay, may also be necessary:

- What is the absolute value of end-to-end delay requirement? (e.g., the ITU-T recommends round trip delays of less than 250msec for telephone calls)

- What is the average throughput and the peak throughput?

- What delay jitter is tolerable?

- Is the communications interactive or in a broadcast mode?

To simplify matters, the discussion below assumes that the throughput achievable in a spectrum hole is affected only by the characteristics of the spectrum hole and the bandwidth available. In practice, the throughput and properties of the available communications channel is also affected by the competing users, the MAC protocol used to resolve contention and the upper layer protocols used.

We further posit that, for a secondary user, communications takes place across a collection of nodes that are separated in space. For successful communications, a spectrum hole must coincide both spatially and temporally with the communicating nodes for the period of the communication. If interference with the licensed user is to be avoided, the radiated signal energy of the secondary user must be substantially contained within the spectrum hole. Thus, the antenna directionality and the location of the radiating nodes make a difference in how a spectrum hole might be used. If a secondary user knew the contours of the spectrum hole, its utility could be maximized through careful system design. However, building a representation of the contours of a spectrum hole would require specific context acquisition approaches (see, e.g., [12]). 
We will organize the remaining discussion according to the temporal characteristics of spectrum holes.

\section{Static Time Spectrum Holes}

The simplest kind of spectrum hole to address is one that is static in time. The main questions that must be addressed by the secondary user for these kinds of spectrum holes is whether the available bandwidth is sufficient for the communications need and whether the spatial configuration of the nodes can be contained within the contours of the spectrum hole. It is possible to have two kinds of (spatial) spectrum holes, contiguous and non-contiguous. The distinction between the two can only be addressed in the context of a particular spatial arrangement of communications nodes.

\section{A. Contiguous space}

In many senses, this represents the easiest type of spectrum hole to use. The main questions that communications engineers have to address with this configuration are:

- Is the available bandwidth sufficient to meet the throughput requirement?

- Are all nodes that must communicate within the boundaries $^{2}$ of the spectrum hole?

If the answers to the above are both affirmative, the spectrum hole can be used for the communication requirement.

The investment that will be required for such a system depends on (1) the required spectral efficiency and (2) whether more sophisticated antennas are required to ensure that the secondary user's signal energy is contained within the spectrum hole. If a node is located near the edge of the spectrum hole, and if the communication is bi-directional, then it may be necessary to use a directional antenna that is generally more costly than an omnidirectional one.

\section{B. Non-contiguous space}

The practicality of this kind of spectrum hole depends on whether transmission paths exist between the spectrum holes in the non-contiguous spatial matrix.

For example, the white regions in the bottom right quadrant of are the result of a shadowing from a series of geological ridges to the west of the town of Kiowa, Colorado. In these holes, only nodes placed within each hole (white areas in Figure 2) would be able to communicate via the $162-174 \mathrm{MHz}$ band modelled in the figure; communications between spectrum holes could not be achieved in this frequency band. Communications between holes must occur out-of-band, possibly at additional cost ${ }^{3}$. The same would be true for noncontiguous spectrum holes that are separated by, for example, low power transmitters, since transmitting through the

\footnotetext{
${ }^{2}$ Note that the nodes as well as their transmission radii must reside within the spectrum hole to prevent interference.

${ }^{3}$ Out-of-band communications channels may occur via a spectrum hole at a different frequency that is not affected by the boundary mechanism; for example, in the situation illustrated in , a spectrum hole at a lower frequency may be able to overcome the geographic barrier that creates the boundary between the holes. Additional cost would be incurred if, for example, a commercial service would have to be purchased to maintain the needed connectivity.
}

occupied spectrum at the operating frequency could result in interference with licensed users.

Thus, the utility of this spectrum hole would be limited to a particular spatial configuration; secondary users requiring a less limited one that exceeds the boundaries of the spectrum hole would incur a higher cost.

Since the spectrum hole is static in time, it would be possible to support elastic and inelastic applications. Any applications would be limited in throughput, of course, by the bandwidth of the spectrum hole ${ }^{4}$.

\section{Periodic Time Spectrum Holes}

Periodic spectrum holes with different origins (and therefore different characteristics) exist. In [1], Weiss et.al. differentiated between periodic and fast periodic spectrum holes, where the latter are those with a period shorter than the context acquisition time; fast periodic spectrum holes might occur in TDMA systems. Fast periodic spectrum holes can only be discovered through cooperation with the primary user; thus, they would be well defined and users could align their communications requirements with them explicitly. Consequently, for the purposes of this paper, periodic spectrum holes are those that can be sensed and used.

Rotating antenna radar is an example of this kind of spectrum hole. In this kind of system, the spectrum hole changes jointly in time and space but in a very predictable way. When the researchers in [13] measured a Terminal Doppler Weather Radar (TDWR) signal at a fixed point in space over time, the periodicity of the radar signal became apparent (20sec) as shown in Figure $4^{5}$. Also apparent was the signal energy in the side lobes.

Another example of periodic time spectrum hole can be inferred from Figure 1. In this figure, the transmitters in several bands are off for distinct times of day (for example, the $614-620 \mathrm{MHz}$ band between approximately 0100 and 0500). If this temporal spectrum trace repeats daily, then the resulting spectrum hole would have contiguous space if all communications nodes were located in the area covered by that television channel.

From a communications perspective, the questions are: What is the minimum delay that could be expected? What average throughput can be expected?

\section{A. Rotating antenna radar}

In the context of Figure 4, if the receivers can tolerate interference of $-50 \mathrm{dBm}$, then approximately $20 \%$ of the rotation is taken up by the main lobe when the antenna elevation is low $^{6}$. After the $10^{\text {th }}$ rotation period, $100 \%$ of the transmission time has signal energy below this threshold. In the $2^{\text {nd }}$ and $3^{\text {rd }}$ rotations, the side lobe energy is sufficiently high to restrict the usable bandwidth to approximately $40 \%$ of

\footnotetext{
${ }^{4}$ The survey that resulted in indicates that the nominal bandwidth of the spectrum hole is $12 \mathrm{MHz}$. If a wideband measurement apparatus were placed in the spectrum hole, the available bandwidth could be larger.

${ }^{5}$ The decreasing peak signal strength in the figure occurs because the elevation of the radar changes over time. Thus, the fixed measurement apparatus would increasingly fall outside of the main lobe of the radar beam.

${ }^{6}$ These figures are estimates derived from careful visual inspection of Figure 4.
} 
the 20 second rotational period.

In summary, for the entire volume 360 second scan pictured in Figure 4, approximately 321 seconds (or $89 \%$ ) would be usable (this decreases if the interference tolerance of the receivers is lower) ${ }^{7}$. In the worst case, the latency would be approximately 8 seconds due to main- and side-lobe interference. This would prohibit inelastic communications, removing that application as a reasonable use case for this kind of spectrum hole (unless substantial buffering is feasible).

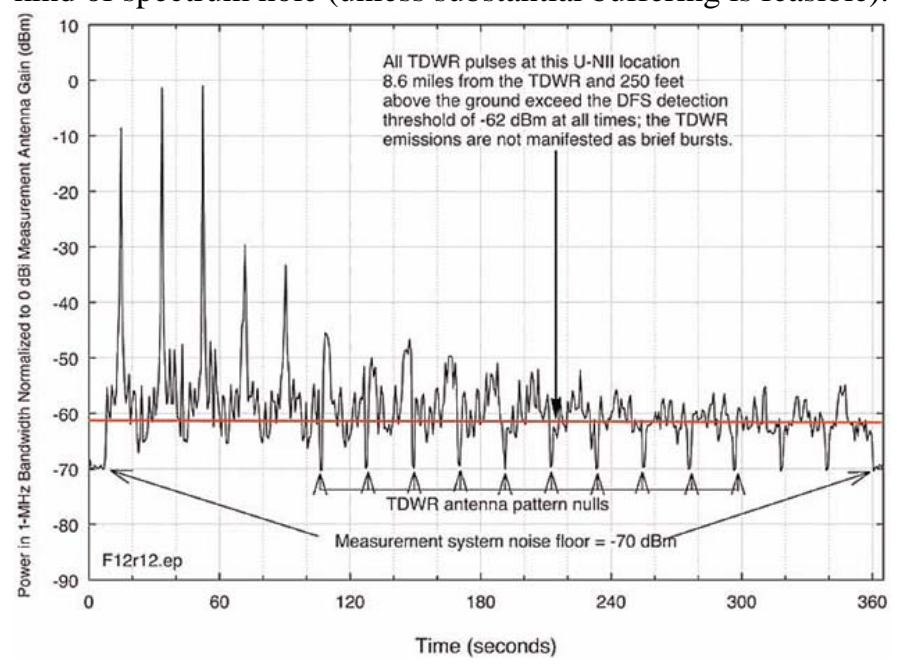

Figure 4 -- Fixed location time domain trace of a radar signal [13]

Rotating antenna radars bifurcate the transmission space at any point in time with the main lobe of their antenna. Thus, it may temporarily create non-contiguous space if the main lobe of the radar separates the communicating nodes. Maintaining communication among the nodes will require out-of-band channels at additional cost.

\section{B. Broadcast transmitter downtime}

Referring to Figure 1, inelastic and elastic communications would clearly be possible during the 0100 to 0500 period in some of the channels depicted. Communications outside of that period would need to use a channel outside of that band. The periodic bifurcation that is characteristic of rotating antenna radars would not apply in this case. Therefore, as long as all nodes were located in the spectrum hole, the spectrum hole would be contiguous.

\section{Stochastic Time Spectrum Holes}

The situation of stochastic time is the most challenging of all. The utility of the spectrum hole depends entirely on the spatial and temporal statistics of the channel with regard to the communications need.

\section{A. Spectrum hole contains user's nodes}

The simpler case occurs if the communications requirement is temporally short and spatially small with respect to the statistics of the spectrum hole. In that case, the secondary user can compute the probability that their communication requirement will be met (assuming that the spatial and temporal distributions of the spectrum hole are known). If the probability is sufficiently high, the secondary user would use the spectrum hole instead of an alternative.

A more complex case occurs when the communication requirement is, with sufficiently high probability, longer than the duration of the spectrum hole. If the secondary user's transmission requirement is elastic, then it could be feasible to wait until the next spectrum hole occurs in the required location. If the resulting average delay is too long, then the secondary user would have to either find an alternative spectrum hole (i.e., on another frequency) or find an alternative communications mechanism (e.g., unlicensed). In this case, the density of spectrum holes across frequency, space and time become important. A high spectrum hole density means that, with sufficiently high probability, the user could move to a new spectrum hole as the availability of the old hole expired. If the density is sufficiently high, then the secondary users could, with a computable probability, transmit at their required QoS, assuming their radios were sufficiently agile, of course.

\section{B. Spectrum hole does not contain the user's nodes}

If the spectrum hole does not (spatially) contain the secondary user's nodes (with sufficiently high probability), then the problem changes again. If the secondary user's communications requirement is sufficiently short such that the probability of successful transmission is sufficiently high, then the secondary user must either (1) find another spectrum hole at another frequency to connect the remaining nodes (2) buffer transmissions to the nodes not contained by the spectrum hole until they can be connected or (3) find an out-of-band mechanism (e.g., unlicensed) to complete the communications. Approach (2) - and possibly also (3) - may only be viable if the secondary user's QoS requirement permits it.

If the secondary user's communication requirement is long with respect to the temporal characteristics of the spectrum hole, the density of the spectrum holes becomes important as described above. However, the problem becomes more complex as the user must manage spectrum holes in both time and space.

\section{Meteor Burst Communications and DSA}

To anticipate the performance that might be expected from a system using stochastic spectrum holes, it is useful to consider an analogous system where the channel availability is exogenously stochastic. Meteor burst communications (MBC) systems (see [14] for an overview) is such a system. Because MBC was studied in some depth and because systems were built, we might get some insight into the system requirements of the kinds of stochastic channels presented by DSA by examining the reported performance of MBC systems.

MBC systems were developed for Beyond Line-of-Sight (BLOS) communications and depend on the statistical frequency of meteors entering the earth's atmosphere RF energy can be reflected off of the ionized trails of these entering meteors to support BLOS communications at

\footnotetext{
${ }^{7}$ The red line in the figure is the Dynamic Frequency Selection (DFS) detection threshold of the U-NII devices in the band being studied.
} 
relatively low cost and with relatively low delay8. According to [14], $10^{10}$ meteors of all sizes enter the earth's atmosphere in a typical $24 \mathrm{hr}$. period and the entry statistics are Poisson. The spatial characteristics of meteor entry are also statistical [15]. These spatio-temporal properties combine to form the duty cycle ${ }^{9}$ of MBC systems. In [14], the duty cycle was measured to be less than $10 \%$ in the systems that they studied.

In order to estimate the impact of duty cycle on communications systems further experiments were performed. The experiments reported round trip times in the worst-case scenario ranging from 50 to 140 seconds and approximately 20 to 60 seconds in the best case scenario. Further, the standard deviations for each collected data point were quite high (26 for the 50 second data point in the worst case trace, for example). Subsequent technological improvements produced better outcomes (see, for example, [16]), but these systems never achieved widespread acceptance, in large measure because of the availability of better alternatives.

\section{Lessons of MBC systems for DSA}

Not surprisingly, the MBC experiments show is that users can expect widely varying QoS that depends on the spatial and temporal statistics of the meteors they rely on. DSA systems rely on spectrum holes instead of meteor trails to facilitate communications, and, in the section we are considering here, these spectrum holes are stochastic and (presumably) exogenous. As with MBC systems, it is of critical importance to understand the spatio-temporal statistics of meteor trails as they affect MBC communications. By analogy, it is of critical importance for secondary users to understand the spatiotemporal statistics of spectrum holes if they can be expected to invest in systems to exploit them. In fact, given the results from [14], a reasonable proposition to advance is that the spatial and temporal statistics of MBC systems set a kind of lower bound for what would be required by secondary users in DSA systems.

Since potential secondary users are directly affected by the characteristics of spectrum holes, it would be unreasonable to expect their active participation in DSA systems of this kind without such a detailed understanding. Though the research in [5] did not assume exogenous spectrum holes, the choice model of the secondary user can be applied here. In [5], the potential secondary user chooses between licensed, unlicensed and secondary use; secondary use is chosen only when the price and QoS preferences dominate the alternatives. When the spatio-temporal statistics of spectrum holes are exogenous, then price falls away as a decision parameter and it becomes a matter of satisfying the potential secondary user's QoS requirement. If the statistics are unknown, then the expected QoS cannot be computed so secondary use (e.g., DSA) would always be dominated by the alternatives.

\section{E. On the Exogeneity of Spectrum Holes}

But it is clear that analogies between MBC and DSA require that statistics of spectrum holes be exogenous. DSA differs from $M B C$ in this regard because spectrum holes need

\footnotetext{
${ }^{8}$ The ion trails of meteors occur at about $90-100 \mathrm{~km}$ altitude, much lower than ionospheric communication or geostationary satellite communications.

${ }^{9}$ The duty cycle is defined as the expected percentage of time from which usable signals can be received and decoded.
}

not be exogenous. For example, the spectrum holes in Figure 1 and could be treated exogenously without loss of generality. In the case of the latter figure, for example, the spectrum holes are due to geological features that are indeed exogenous.

However, if one considers the case of the spectrum holes that occur from 0100 to 0500 in Figure 1, the situation is less clear. These spectrum holes would be exogenous if the licensed user chose to turn off their transmitters during this period independent of any potential secondary use of this spectrum. For example, in [17], the authors propose turning off unused radios during periods of slack demand as part of an optimal energy savings strategy

But such spectrum holes need not be exogenous. When cooperative sharing is considered, spectrum holes may be the result of bargaining on the part of primary and secondary users. To illustrate, let us reconsider the dimming strategy proposed in [17]: if the mobile operator's computation included potential revenues from secondary user, the primary users could become more aggressive in shutting down radios, thereby creating spectrum holes that could be used by secondary users. Endogenously derived spectrum holes would be an outcome most likely to occur in these cooperative scenarios as was suggested by [18] as a rational strategy for operators; these cooperative scenarios were modelled by [5] and [19] among others.

The main conclusion here is that if spectrum holes are endogenous, then alternate tools should be used to characterize them, as their arrival rate cannot legitimately be considered as occurring from a Poisson process.

The question of the density of spectrum holes that was raised in the previous section, then, should be addressed by considering spectrum holes both as exogenous as well as endogenous phenomena. Like Swiss cheese, the density of spectrum holes is the result of natural (i.e., exogenous) and manufactured (i.e., endogenous) inputs.

For secondary users, this means that they are partially masters of their own fate. If the communications requirement of secondary users is sufficiently valuable and if the exogenous spectrum hole density is not sufficient, then the secondary user would be motivated to share the benefits of the communication by bargaining with primary users to create the needed spectrum holes. In other words, if they don't find what they need, they may have to invest to make it.

\section{SUMMARY}

The previous sections have considered which secondary users' communications might be met using DSA-based systems. The conclusions of this analysis are collected and summarized in Figure 5.

- It is clear that inelastic communications requirements can only be supported without reservation by static, contiguous spectrum holes. Other kinds of spectrum holes require either a secondary channel to connect non-contiguous nodes or are limited to uni-directional channels with buffering. In the case of stochastic spectrum holes, the ability to support inelastic secondary user's communications depends entirely on the density of the spectrum holes. 


\begin{tabular}{|c|c|c|c|}
\hline & \multicolumn{2}{|c|}{ Spatial Characteristic } \\
\hline & & Contiguous & Non-Contiguous \\
\hline \multirow{3}{*}{ 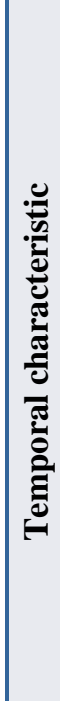 } & 胥 & $\begin{array}{ll}\text { - } & \text { Elastic \& inelastic } \\
\text { applications } \\
\text { - } & \text { Broadcast }\end{array}$ & $\begin{array}{ll}\text { - } & \text { Additional cost to } \\
\text { connect bifurcated } \\
\text { networks } \\
\text { - } & \text { Elastic \& inelastic } \\
\text { - } & \text { Broadcast }\end{array}$ \\
\hline & 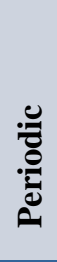 & $\begin{array}{ll}\text { - } & \text { Elastic } \\
\text { - } & \text { Inelastic w. limits } \\
\text { - } & \text { Broadcast with } \\
\text { - } & \text { Buffay } \\
\text { broadcast }\end{array}$ & $\begin{array}{ll}\text { - } & \text { Additional cost to } \\
\text { connect bifurcated } \\
\text { networks } \\
\text { - } & \text { Elastic } \\
\text { - } & \text { Inelastic w. limits }\end{array}$ \\
\hline & 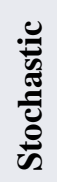 & $\begin{array}{l}\text { - } \quad \text { Elastic } \\
\text { - Inelastic only with } \\
\text { sufficient "hole } \\
\text { density" }\end{array}$ & $\begin{array}{ll}\text { - } & \text { Additional cost to } \\
\text { connect bifurcated } \\
\text { networks } \\
\text { - } & \text { Elastic }\end{array}$ \\
\hline
\end{tabular}

Figure 5 -- Summary of application characteristics by spectrum hole type

Little is known about the density of spectrum holes across frequency, space and time. Some work has been performed to study spectrum holes based on a primary user's activities [20], but it remains focussed on a single band and does not yet help a potential secondary user determine if their communications requirements can be met using DSA. Clearly, this is an area requiring considerably more research.

\section{CONCLUSION AND FURTHER RESEARCH}

In bilateral systems such as DSA, it is necessary to consider the decision processes of both parties (i.e., the primary and secondary users). Failure to do so can limit practical applications of these technologies. It is important for the emergent regulatory policies to explicitly consider the requirements of secondary users, especially since they are generally not well represented in regulatory proceedings because of the structural aspects of the emergent market. In other words, secondary users do not yet exist in meaningful numbers, nor is the optimal application of this technology clear yet, so their interests cannot be easily articulated in regulatory proceedings.

In this paper I argue that one approach to address this imbalance is to explicitly consider the impact of the spatiotemporal properties of spectrum holes on the use decision by potential secondary users. While my analysis was largely qualitative, its main objective was to set the stage for more careful analytical analyses in future.

This paper also makes clear the importance of studying and modeling the spatial and temporal properties of spectrum holes in greater detail. This is especially the case as one moves away from spectrum holes that are static in time and space. In particular, a research campaign is necessary that will characterize the temporal and spatial properties of spectrum holes. This research must extend the work of [20] [21] to first model and then measure spectrum holes across frequency bands. This research must also consider the question of exogeneity of spectrum holes.

Developing usable characterizations of spectrum holes in frequency, time and space is necessary. These characterizations must be explicitly linked to user communications requirements and the spatio-temporal characteristics of spectrum holes. This is clearly an area of research that will require considerable development.

General application of DSA will require not just the spatial and temporal characteristics of spectrum holes, but also the density of spectrum holes across numerous frequency bands to determine if (1) out of band communications between noncontiguous spectrum holes is possible without purchasing commercial services and (2) if inelastic user requirements can be supported.

\section{REFERENCES}

M. B. H. Weiss, et al., "Dynamic Geospatial Spectrum Modelling: Taxonomy, Options and Consequences," presented at the Telecommunications Policy Research Conference, Arlington VA, 2010. dynamic spectrum assignment," Pittsburgh PA, Working Paper http://d-scholarship.pitt.edu/2824/, 2009.

I. F. Akyilidiz, et al., "NeXT generation/dynamic spectrum access/cognitive radio wireless networks: A survey," Computer Networks, vol. 50, pp. 2127-2159, 2006.

J. M. Chapin and W. H. Lehr, "The path to market success for dynamic spectrum access technology," IEEE Communications Magazine, vol. 45, pp. 96-103, May 2007.

A. Tonmukayakul and M. B. H. Weiss, "A study of secondary spectrum use using agent-based compuational economics," Netnomics, vol. 9, pp. 125-151, 2008.

R. Tandra, et al., "What is a Spectrum Hole and What Does It Take to Recognize One?," Proceedings of the IEEE, vol. 97, May 2009. J. E. Carroll, et al., "Measurements to Characterize Land Mobile Channel Occupancy for Federal Bands 162-174 MHz and 406-420 $\mathrm{MHz}$ in the Denver CO Area," National Telecommunication and Information Administration2008.

M. Wellens, et al., "Spatial statistics and models of spectrum use," Computer Communications, vol. 32, pp. 1998-2011, Dec 152009. P. Mähönen, et al., "Applications of Topology Information for Cognitive Radios and Networks," in New Frontiers in Dynamic Spectrum Access Networks, 2007. DySPAN 2007. 2nd IEEE International Symposium on, 2007, pp. 103-114.

J. Riihijärvi and P. Mähönen, "Exploiting Spatial Statistics of Primary and Secondary Users towards Improved Cognitive Radio Networks," in Cognitive Radio Oriented Wireless Networks and Communications, 2008. CrownCom 2008. 3rd International Conference on, 2008, pp. 1-7.

W. Stallings, Data and Computer Communications, 9th ed. Upper Saddle River, NJ: Prentice-Hall, 2011.

M. B. Weiss, et al., "Sensing as a Service: An exploration into the practical implementation of DSA," in IEEE DySPAN, Singapore, 2010.

J. E. Carroll, et al., "Case Study: Investigation of Interference into $5 \mathrm{GHz}$ Weather Radars from Unlicensed National Information Infrastructure Devices, Part I," Institute for Telecommunications Sciences, US Dept of Commerce, NTIA, Boulder CO TR-11-473, 2010.

D. Yavuz, "Meteor burst communications," IEEE Communications Magazine, vol. 28, pp. 40-48, Sept 1990. 
[15] A. N. Ince, "Spatial Properties of Meteor-Burst Propagation," IEEE Transactions on Communications, vol. COM-28, pp. 841849, June 1980.

[16] R. S. Mawrey, et al., "Conversational voice communication over a high performance meteor burst channel," in Military Communications Conference, 1992, pp. 18-24.

[17] D. Tipper, et al., "Dimming Cellular Networks," in IEEE Globecom, Miami Florida, 2010.

[18] J. M. Peha and S. Panichpapiboon, "Real-time secondary markets for spectrum," Telecommunications Policy, vol. 28, pp. 603-618, 2004.

[19] C. Caicedo and M. B. Weiss, "The viability of secondary spectrum trading markets," in Proceedings of IEEE DySPAN, Singapore, 2010.

[20] J. Riihijärvi, et al., "Impact of primary user activity patterns on spatial spectrum reuse opportunities," in Wireless Conference (EW), 2010 European, 2010, pp. 962-968.

[21] J. Riihijärvi and P. Mähönen, "Modeling Spatial Structure of Wireless Communication Networks," in INFOCOM IEEE Conference on Computer Communications Workshops, 2010, pp. 1-6. 